\title{
Magnesium: Bedeutung für die hausärztliche Praxis - Positionspapier der Gesellschaft für Magnesium-Forschung e. V.
}

\section{Magnesium: Relevance for general practitioners - a position paper of the Society for Magnesium Research e. V.}

Autoren

Oliver Micke ${ }^{1}$, Jürgen Vormann², Hans-Georg Classen ${ }^{3}$, Klaus Kisters ${ }^{4}$

\section{(ㄷ) (i) $(9)$}

Institute

1 Klinik für Strahlentherapie und Radioonkologie, Franziskus Hospital Bielefeld

2 Institut für Prävention und Ernährung, Ismaning

3 Ehemals FG Pharmakologie und Toxikologie der Ernährung, Universität Stuttgart-Hohenheim

4 Medizinische Klinik I, St.-Anna-Hospital Herne

Schlüsselwörter

Magnesium, Magnesiummangel, Hypomagnesiämie, kardiovaskuläre Erkrankungen, Diabetes mellitus

Key words

magnesium, magnesium deficiency, hypomagnesemia, cardiovascular diseases, diabetes mellitus

\section{Bibliografie}

Dtsch Med Wochenschr 2020; 145: 1628-1634

DOI 10.1055/a-1166-7229

ISSN 0012-0472

\section{(c) 2020. The Author(s).}

This is an open access article published by Thieme under the terms of the Creative Commons Attribution-NonDerivative-NonCommercial License, permitting copying and reproduction so long as the original work is given appropriate credit. Contents may not be used for commecial purposes, or adapted, remixed, transformed or built upon. (https://creativecommons.org/licenses/by-nc-nd/4.0/)

Korrespondenzadresse

Prof. Dr. med. Oliver Micke

Franziskus Hospital Bielefeld, Klinik für Strahlentherapie und Radioonkologie, Kiskerstraße 26, 33615 Bielefeld, Deutschland

Tel.: ++ 49/521/5891801

Fax: $++49 / 521 / 5891804$

oliver.micke@franziskus.de

\section{ZUSAMMENFASSUNG}

In der Bevölkerung und vor allem bei Risikogruppen muss mit Magnesiummangel gerechnet werden. Magnesiummangel kann zahlreiche Symptome verursachen, ist per se pathologisch und deshalb therapiebedürftig. Die Diagnostik stützt sich auf die klinische Symptomatik in Verbindung mit anamnestischen Hinweisen und Laborparametern. Eine schlechte Magnesiumversorgung ist mit einem erhöhten Risiko für zahlreiche Erkrankungen assoziiert, darunter metabolisches Syndrom, Diabetes mellitus Typ 2 und Herz-Kreislauf-Erkrankungen. Magnesiummangel tritt häufig auch als Komorbidität auf und kann zur Exazerbation von Krankheiten beitragen. Magnesium sollte eine größere Aufmerksamkeit geschenkt werden, um Mangelzustände als Ursache vielfältiger Symptome sowie als Risikofaktor für Erkrankungen zu vermeiden. Eine Optimierung der Magnesiumversorgung kann einen wichtigen Beitrag in der Prävention leisten. Die orale Magnesiumtherapie ist unbedenklich und kostengünstig.

\section{ABSTRACT}

Magnesium deficiency is to be expected in the population and particularly among risk groups. Magnesium deficiency can cause numerous symptoms, is per se pathological and thus requires treatment. Diagnostics is based on clinical symptoms in conjunction with anamnestic criteria and laboratory parameters. Insufficient magnesium supply is associated with an increased risk for many diseases, e. g. metabolic syndrome, type 2 diabetes and cardiovascular diseases. Magnesium deficiency often appears as comorbidity and may exacerbate diseases. Physicians should pay more attention to magnesium in order to avoid deficits as a cause for multiple symptoms and risk factor for diseases. Optimisation of magnesium status may make an important contribution to the prevention of diseases. Oral magnesium therapy is safe and cost effective. 
- Tab. 1 Mögliche Symptome eines Magnesiummangels (Auswahl) [2, 16].

\begin{tabular}{|c|c|}
\hline Bereich & Anzeichen/Symptome \\
\hline allgemeiner Bereich & $\begin{array}{l}\text { Schwäche, Lethargie, Müdigkeit, Schlafstörungen, reduzierte Stresstoleranz, verschlechterte sportliche Leistung, } \\
\text { Agitiertheit, Reizbarkeit, Hyperaktivität, Chronic-Fatigue-Syndrom }\end{array}$ \\
\hline neuromuskulärer Bereich & $\begin{array}{l}\text { Hyperreflexie, Muskelkrämpfe (Waden, Fußsohlen, Beine, Gesicht), Muskelspasmen/Karpopedalspasmen, Muskel- } \\
\text { schwäche, Nacken-/Rückenschmerzen, Magnesiummangeltetanie, Nystagmus, Tremor, Faszikulationen, positives } \\
\text { Chvostek-Zeichen, positiver Trousseau-Test }\end{array}$ \\
\hline Nervensystem & $\begin{array}{l}\text { Nervosität, Übererregbarkeit, Kopfschmerzen/Migräne, Depression, Aggressionen, Angststörungen/Psychosen, } \\
\text { Verwirrtheit, Desorientiertheit, verringerte Gedächtnisleistung, Schwindel, Krampfanfälle, Parästhesien (Taubheits- } \\
\text { gefühle, Kribbeln etc.), erhöhte Sensitivität des NMDA-Rezeptors auf exzitatorische Neurotransmitter, Hyperalgesie, } \\
\text { Ataxien, Hörverlust }\end{array}$ \\
\hline Gastrointestinaltrakt & Obstipation, Übelkeit, Spasmen \\
\hline kardiovaskuläres System & $\begin{array}{l}\text { Herzrhythmusstörungen (ventrikuläre und supraventrikuläre Arrhythmien, Vorhofflimmern, Torsade-de-pointe), } \\
\text { Hypertonie, Arteriosklerose, Koronarspasmen, reduzierte myokardiale Pumpfunktion, erhöhte Digitalis-Sensitivität, } \\
\text { verstärkte Thrombozytenaggregation, EKG-Veränderungen (u. a. verlängertes QT-Intervall, ST-Senkung) }\end{array}$ \\
\hline Elektrolytstörungen & Hypokaliämie, Hypokalzämie, Natriumretention \\
\hline Stoffwechsel & $\begin{array}{l}\text { Insulinresistenz, reduzierte Glukosetoleranz, erhöhtes Risiko für metabolisches Syndrom, gestörte Bildung von } \\
\text { aktivem Vitamin D, Vitamin-D-Resistenz, gestörte Parathormonfreisetzung, Parathormonresistenz, Dyslipoprotein- } \\
\text { ämie (Hypertriglyzeridämie, Hypercholesterinämie) }\end{array}$ \\
\hline gynäkologischer Bereich & vorzeitige Wehen, Abortneigung, (Prä-) Eklampsie, Dysmenorrhoe \\
\hline verschiedenes & Asthma, Osteoporose, Neigung zu Kalzium-Oxalat-Nierensteinen, Kalzifikation von weichem Gewebe \\
\hline
\end{tabular}

\section{Hintergrund}

Magnesium (Mg) ist an mehr als 600 enzymatischen Reaktionen beteiligt, fungiert als physiologischer Kalziumantagonist und spielt als Bestandteil von ATP in praktisch allen zellulären Prozessen eine Rolle [1, 2].

\section{Merke}

Die Mg-Mangelsymptomatik präsentiert sich vielfältig, da Magnesium an einer Vielzahl von physiologischen Prozessen beteiligt ist ( $\triangleright$ Tab. 1$)$.

Ziel der Übersicht ist es, Mg-Mangelzustände rechtzeitig zu erkennen und adäquat zu therapieren, um sie als Ursache von Symptomen sowie als Risikofaktor für Erkrankungen zu vermeiden.

\section{Methoden}

In der Datenbank PubMed wurde eine Literaturrecherche mit dem Suchbegriff Magnesium durchgeführt: Zeitraum: 01.01.200015.12.2019; Volltext in englischer oder deutscher Sprache. Des Weiteren wurden Studienzitate in den aufgefundenen Publikationen und Veröffentlichungen der Gesellschaft für MagnesiumForschung (www.magnesium-ges.de) herangezogen.

\section{Kritische Versorgungssituation}

In Deutschland nehmen 29\% der Frauen und 26\% der Männer weniger Mg über die Nahrung zu sich als für die gesunde Bevölkerung empfohlen wird. Bei den 14-18-jährigen Mädchen ist der
Anteil mit $56 \%$ besonders hoch $(n=15371)$ [3]. In der VERAStudie ( $n=2006)$ wurde bei $10 \%$ der Erwachsenen eine deutliche Hypomagnesiämie festgestellt; bei den 18-24-jährigen Frauen betrug der Anteil $20 \%$ [4]. Die hier festgelegten Grenzwerte (Frauen: Serum-Mg < 0,67; Männer: <0,69 mmol/l) erscheinen dabei bemerkenswert niedrig (siehe Diagnostik). In einer weiteren Stichprobe $(n=16017)$ lag die Prävalenz der Hypomagnesiämie $(<0,76 \mathrm{mmol} / \mathrm{l})$ bei $14,5 \%$. Suboptimale Versorgungszustände $(<0,80 \mathrm{mmol} / \mathrm{l})$ wurden bei 33,7\% gefunden [5]. Ähnliche Daten wurden auch in anderen westlichen Ländern registriert [6]. Insofern muss in der Allgemeinbevölkerung mit Mg-Mangelzuständen gerechnet werden. In Risikogruppen wurden zum Teil deutlich höhere Hypomagnesiämieprävalenzen gefunden, z. B. Diabetiker (bis $48 \%$ ) [7], ältere Menschen (33\%) [8], Sportler (>50\%) [9], Schwangere (72\%) [10], Alkoholiker (30\%) [11] sowie kritisch Kranke (bis $65 \%$ ), assoziiert mit erhöhter Mortalitätsrate und längeren Klinikaufenthalten [12].

\section{Merke}

Ein Viertel der Deutschen nimmt über die Nahrung weniger Magnesium zu sich als empfohlen wird.

\section{Problematische Diagnostik}

Eine Hypomagnesiämie ist ein deutliches Zeichen für einen Mg-Mangel; Normalwerte schließen Mangelzustände jedoch nicht aus [13]. Grund dafür ist, dass sich im Plasma nur 0,3\% des gesamten $\mathrm{Mg}$ befinden und das Plasma-Mg durch Freisetzung aus Geweben lange aufrechterhalten wird. So deckt der sensitive Mg-Retentionstest Mangelzustände auf, die anhand des Serum- 
- Tab. 2 Hinweise und Empfehlungen zur Bestimmung des Serum-Mg.

allgemeine Hinweise

Serum wird gegenüber Plasma bevorzugt (zur Plasmagewinnung nötige Antikoagulanzien können Magnesium komplexieren)

zirkadiane Rhythmik und Körperposition bei der Blutentnahme können das Serum-Mg beeinflussen
Blutabnahme

wenn möglich, keine Blutstauung bzw. nur leicht und kurz stauen (Gefahr der Hämolyse)

möglichst schonende Blutabnahme; Aspirationssysteme sind gegenüber Unterdrucksystemen im Vorteil
Serumgewinnung

kein Schütteln oder starkes Abkühlen von Vollblut

idealerweise Abzentrifugation des Serums vor Ort:

- Vollblut mindestens 20 min, max. 60 min vor der Zentrifugation aufbewahren (Röhrchen sollten stehen, nicht liegen)

- sachgerechte Zentrifugation (10 min bei $2000 \mathrm{~g}$ mit

freischwingender Zentrifuge)

- Serum vorsichtig in Versandgefäß pipettieren (nicht umgießen, da hiermit Erythrozyten mitgerissen werden)

Erkennen und Vermeiden von Stresssitua-

tionen bei der Blutabnahme

Mg nicht erkennbar sind. Deshalb wird die Prävalenz des Mg-Mangels anhand des Serum-Mg unterschätzt. Aussagekräftigere Parameter stehen in der Standarddiagnostik nicht zur Verfügung (z. B. ionisiertes $\mathrm{Mg}$ im Serum, Lymphozyten- oder Muskel-Mg, $\mathrm{Mg}$-Retentionstest). Vorgeschlagen wird ein Referenzbereich von 0,76-1,10 mmol/l (1,85-2,67 mg/dl) [14], wobei aus präventiver Sicht $>0,85 \mathrm{mmol} / \mathrm{l}$ anzustreben sind [15]. Im Leistungsverzeichnis von Laboren finden sich z. T. deutlich zu niedrige Grenzwerte, z. B. $0,66 \mathrm{mmol} / \mathrm{l}$.

\section{Cave}

Normale Magnesiumkonzentrationen im Blut schließen Mangelzustände nicht aus. Bei Hämolyse besteht die Gefahr falsch hoher Serum-Mg-Werte ( $\triangleright$ Tab. 2).

Die Diagnostik des Mg-Mangels besteht somit aus den Säulen Mangelsymptomatik ( Tab. 1), Labordiagnostik und Risikofaktoren ( $\triangleright$ Tab.3). Aufgrund der großen Bedeutung des Mg-Mangels (und des geringen Kostenaufwands) sollte das Mg bei den SerumElektrolyten routinemäßig mitbestimmt werden.

\section{Klinische Aspekte}

Epidemiologische Studien zeigen einen inversen Zusammenhang zwischen Mg-Zufuhr oder Serum-Mg mit dem Auftreten bedeutsamer Erkrankungen, z. B. metabolisches Syndrom bzw. Insulinresistenz, Typ-2-Diabetes, Herz-Kreislauf-Erkrankungen, plötzlicher Herztod und Alzheimer, und der Gesamtmortalität [1, 2, 16, 17]. Mg-Mangel muss deshalb als Risikofaktor in Betracht gezogen werden.

\section{Insulinresistenz, metabolisches Syndrom und Diabetes mellitus}

Aufgrund der Funktionen im Insulin- und Glukosestoffwechsel kommt es bei Mg-Mangel zu einer Insulinresistenz und gestörten Glukosetoleranz - eine plausible Erklärung für die epidemiologische Datenlage, nach der niedrige Mg-Zufuhr bzw. niedriges Serum-Mg mit einem erhöhten Risiko für Insulinresistenz, meta- bolisches Syndrom und Typ-2-Diabetes verbunden ist (z. B. [18]). In randomisierten, kontrollierten Interventionsstudien (RCTs) wurde durch orales $\mathrm{Mg}$ eine Verbesserung der Insulinresistenz erreicht; in der Effektstärke vergleichbar mit Lebensstilinterventionen (z. B. Gewichtsabnahme). Bei Typ-2-Diabetes reduzierte sich durch $\mathrm{Mg}$ die Nüchternglukose [19]. Für eine Senkung des HbA1c waren die meisten Studien zu kurz ( $\leq 12$ Wochen). Mg-Mangel gilt als Risikofaktor für Nephropathie, Polyneuropathie sowie Retinopathie und ist mit erhöhter Mortalität assoziiert. Der Zusammenhang zwischen Mg und Diabetes bzw. Insulinresistenz wurde in zahlreichen Reviews thematisiert (z. B. [20]).

\section{Hypertonie}

Mg-Mangel verursacht über eine gesteigerte Gefäßreaktivität und einen gesteigerten Gefäßtonus einen Blutdruckanstieg.

\section{Merke}

Die blutdrucksenkende Wirkung oraler Mg-Einnahme konnte in mehreren Metaanalysen über RCTs bestätigt werden (z. B. [21]).

In unselektierten Kollektiven war der Effekt moderat (Systole ca. 2-4 mmHg, Diastole ca. -2 mmHg), bei Hypertonikern unter antihypertensiver Therapie jedoch stark ausgeprägt (Systole $19 \mathrm{mmHg}$, Diastole $-11 \mathrm{mmHg}$ ) [21]. Eine blutdrucksenkende Wirkung ist ab etwa $300 \mathrm{mg} \mathrm{Mg}$ pro Tag zu erwarten. Bereits eine Reduktion der Systole um $0,8-2 \mathrm{mmHg}$ ist als klinisch relevant zu erachten. Bei Frauen unter Thiazid-Therapie wurde in einer RCT durch $600 \mathrm{mg}$ Mg täglich über 6 Wochen eine Blutdrucksenkung von 144 auf $134 \mathrm{mmHg}$ bzw. von 88 auf $81 \mathrm{mmHg}$ erreicht [22]. Neben Lebensstiländerungen bietet sich Mg zur Prävention sowie als adjuvante therapeutische Maßnahme an.

Cave

Diuretika verursachen renale Mg-Verluste ( $>$ Tab. 3 ).

\section{Arteriosklerose}

Mg-Mangel fördert proatherogene und prothrombotische Bedingungen. Zu den Mechanismen gehören u. a. verstärkte Entzün- 
- Tab.3 Anamnestische Hinweise auf einen Mg-Mangel [2, 7, 14, 16, 40].

\begin{tabular}{|c|c|c|}
\hline allgemeine Anamnese & Vor-|Begleiterkrankungen & Arzneimittel \\
\hline $\begin{array}{l}\text { Ernährung, z. B. Reduktionsdiätena; magnesium- } \\
\text { arme Lebensmittela (hoher Anteil an verarbeite- } \\
\text { ten bzw. geringer Anteil an naturbelassenen } \\
\text { Nahrungsmitteln); fettreiche Ernährung } \\
\text { phatreiche Ehos- } \\
\text { den, Fast-Food); Unterernährung/Magersucht }{ }^{\mathrm{a}}\end{array}$ & Diabetes mellitus ${ }^{\mathrm{b}, \mathrm{c} ;}$ Insulinresistenz ${ }^{\mathrm{c}}$ & $\begin{array}{l}\text { Schleifendiuretikac (z. B. Furosemid, } \\
\text { Torasemid, Ethycrynsäure); } \\
\text { osmotische Diuretikac (z. B. Mannitol); } \\
\text { Thiaziddiuretika` (z. B. Hydrochlorothiazid, } \\
\text { Chlortalidon, Xipamid) }\end{array}$ \\
\hline Sport bzw. starke körperliche Aktivität ${ }^{c, d, ~ e ~}$ & $\begin{array}{l}\text { Darmerkrankungen }{ }^{\mathrm{b}} \text { (Morbus Crohn, Colitis } \\
\text { ulcerosa, Zöliakie) }\end{array}$ & $\begin{array}{l}\text { Protonenpumpenhemmerb (z. B. Omeprazol, } \\
\text { Esomeprazol, Pantoprazol, Lansoprazol) }\end{array}$ \\
\hline $\begin{array}{l}\text { Schwangerschaft, e; } \\
\text { Stillzeit }^{\mathrm{f}}\end{array}$ & $\begin{array}{l}\text { Durchfall'; } \\
\text { Erbrechen }^{\text {b }}\end{array}$ & $\begin{array}{l}\text { Platinverbindungenc (Cisplatin, Carboplatin, } \\
\text { Oxaliplatin) }\end{array}$ \\
\hline chronischer Stress $^{c}$ & Zustand nach Darmresektion ${ }^{\mathrm{b}}$; Kurzdarmsyndrom ${ }^{\mathrm{b}}$ & EGFR-Inhibitorenc (z. B. Cetuximab, Erlotinib) \\
\hline hoher Alkoholkonsum a, b, c & Zustand nach bariatrischer Chirurgie ${ }^{b}$ & $\begin{array}{l}\text { Aminoglykosid-Antibiotikac (z. B. Amikacin, } \\
\text { Gentamicin, Tobramycin) }\end{array}$ \\
\hline höheres Alter ${ }^{\mathrm{a}, \mathrm{b}}$ & $\begin{array}{l}\text { angeborene Mg-Verlusterkrankungen (z. B. Gitel- } \\
\text { mansyndromc; familiäre Hypomagnesiämie mit } \\
\text { Hyperkalzurie und Nephrokalzinose (FHHNC); } \\
\text { Hypomagnesiämie mit sekundärer Hypokalzämie } \\
(\mathrm{HSH})^{\mathrm{b},(\mathrm{c})} \text {; Bartter-Syndrom Typ 3c) }\end{array}$ & $\begin{array}{l}\text { weitere antimikrobielle Substanzenc } \\
\text { (Pentamidin, Amphotericin B, Foscarnet) }\end{array}$ \\
\hline \multirow[t]{6}{*}{ Mg-Mangelsymptome in der Familienanamnese } & $\begin{array}{l}\text { Pankreatitis } \\
\text { Steatorrhoe }^{\mathrm{b}}\end{array}$ & $\begin{array}{l}\text { Immunsuppressivac (Cyclosporin A, } \\
\text { Tacrolimus, Sirolimus (= Rapamycin)) }\end{array}$ \\
\hline & $\begin{array}{l}\text { metabolische Azidose }{ }^{c} \text { (z. B. Hungern, diabetische } \\
\text { Ketoazidose) }\end{array}$ & $\begin{array}{l}\text { Laxantien }{ }^{\text {b }} \text { (Ausnahme: Mg-haltige Laxantien, } \\
\text { z. B. Mg-Sulfat (Bittersalz)) }\end{array}$ \\
\hline & $\begin{array}{l}\text { Syndrom des hungrigen Knochens }{ }^{g} \text {; Refeeding- } \\
\text { Syndrom }^{g}\end{array}$ & Bisphosponate ${ }^{\mathrm{C}}$ (Pamidronat) \\
\hline & $\begin{array}{l}\text { endokrinologische Störungen (Hyperthyreosec; } \\
\text { Hypoparathyreoidismus }^{\mathrm{b}, \mathrm{c} ;} \text {; Hyperaldosteronismus }{ }^{\mathrm{c}} \text { ) }\end{array}$ & Digoxin $^{c}$ \\
\hline & $\begin{array}{l}\text { Nierenerkrankungen` (Pyelonephritis; Glomerulo- } \\
\text { nephritis; diuretische Phase der akuten Tubulusne- } \\
\text { krose; obstruktive Nephropathie; renal-tubuläre } \\
\text { Azidose; Dialyse) }\end{array}$ & Östrogene ${ }^{g}$ \\
\hline & $\begin{array}{l}\text { weitere Ursachen: } \\
\text { Sepsisc; Proteinuriec; Hyperkalzämiec }^{c}\end{array}$ & \\
\hline
\end{tabular}

hauptsächliche Mechanismen: a = unzureichende Zufuhr; b = gastrointestinale Verluste/eingeschränkte intestinale Resorption; $c=$ renale Verluste; $d=$ Verluste über den Schweiß; $\mathrm{e}=$ erhöhter Bedarf; $\mathrm{f}=$ Abgabe über die Muttermilch; $\mathrm{g}$ = verstärkte Umverteilung im Gewebe.

dungstendenz, proatherogenes Lipidprofil, gestörte Endothelfunktion und verstärkter Kalziumeinbau in der Gefäßwand [16]. Epidemiologisch besteht eine inverse Assoziation zwischen $\mathrm{Mg}$ Zufuhr bzw. Serum-Mg und koronarer Herzkrankheit (KHK) sowie peripherer arterieller Verschlusskrankheit $[18,23]$. In RCTs wurden durch orales $\mathrm{Mg}$ günstige Effekte erzielt, u. a. eine Verbesserung der Endothelfunktion [24], ein Progressionsstopp der IMT bei Risikopatienten (z. B. [22]), eine Verbesserung der Gefäßsteifigkeit bei Übergewichtigen [25] und eine Steigerung der Leistungsfähigkeit bei KHK-Patienten (z. B. [26]).

\section{Herzinsuffizienz}

Herzinsuffiziente weisen u. a. aufgrund einer Überstimulierung des Renin-Angiotensin-Aldosteron-Systems und Diuretika-Einnahme häufig Mg-Mangelzustände auf [27], die die Prognose verschlechtern. So wurde bei herzinsuffizienten Patienten mit niedrigem Serum-Mg eine höhere Mortalitätsrate registriert [28]. Es ist deshalb zwingend auf einen optimalen Mg-Status zu achten; insbesondere bei Digitalis-Therapie, da Mg-Mangel dessen Toxizität erhöht [27]. In RCTs konnte durch Mg eine längere Lebensdauer, eine Verbesserung der Lebensqualität und Belastbarkeit sowie eine Reduzierung von Herzrhythmusstörungen erzielt werden [29, 30].

\section{Herzrhythmusstörungen}

Es ist gut bekannt, dass Mg-Mangel zu einer Übererregbarkeit der Herzmuskelzellen und infolge dessen zu Herzrhythmusstörungen führen kann. Verantwortlich dafür ist u. a. ein sekundärer Kalium (K) -Mangel. Bevor Antiarrhythmika eingesetzt werden, sollte deshalb ein Mg-Mangel ausgeglichen werden. Orales Mg besitzt dabei ein ausgesprochen günstiges Nutzen-Risiko-Profil [31]. Pharmakologische Effekte, $d$.h. eine (therapeutisch) induzierte 
Hypermagnesiämie, sind nur durch eine parenterale Mg-Gabe zu erwarten. Zu den Rhythmusstörungen, die hierfür infrage kommen, zählen u. a. multifokale atriale Tachykardie, Vorhofflimmern (vor allem nach Herzchirurgie) und Kammertachykardien [31].

\section{Merke}

Mittel der Wahl ist Mg i. v. bei Torsade-de-pointes-Tachykardien. Sie werden insbesondere durch QT-verlängernde Arzneistoffe ausgelöst (siehe www.crediblemeds.org).

Da Mg- und K-Mangel ebenfalls eine QT-Verlängerung verursachen, müssen vor der Therapie Mangelzustände zwingend ausgeglichen werden. Auf diesen Umstand wird nicht in allen Fachinformationen QT-verlängernder Arzneimittel hingewiesen. Ein K-Mangel lässt sich erst beheben, wenn ein (häufig begleitender) Mg-Mangel beseitigt wird. Selbst wenn das Serum-K ansteigt, kann bei Mg-Mangel ein zellulärer K-Mangel fortbestehen [32]. Internationale Fachgesellschaften haben $\mathrm{Mg}$ zur Prävention und zum Management von Herzrhythmusstörungen in ihren Leitlinien implementiert (z. B. [33]).

\section{Schwangerschaft und Kinderheilkunde}

Die Schwangerschaft gilt als klassische Mg-Mangelsituation. Ursachen sind eine häufig unzureichende Mg-Zufuhr, ein erhöhter Mg-Bedarf durch mütterliches Gewebewachstum und Wachstum des Kindes sowie stoffwechselbedingte renale Mg-Verluste [34]. In den meisten RCTs konnten durch orales Mg positive Effekte erzielt werden, z. B. weniger Spontanaborte und vorzeitige Wehen, Frühgeburtlichkeit, geringeres Auftreten von Hypertonie und Wadenkrämpfen, geringere Hospitalisations- bzw. Verlegungsrate auf Intensivstation [34]. Bei Muskelkrämpfen ist Mg Mittel der Wahl, da das risikoreiche Chinin in Schwangerschaft und Stillzeit kontraindiziert ist.

\footnotetext{
Merke

Aufgrund der präventiven Bedeutung bei fehlendem Risiko wird Schwangeren eine generelle orale Mg-Supplementierung von 240-480 mg (10-20 mmol) täglich empfohlen.
}

Orales $\mathrm{Mg}$ beeinflusst die physiologischen Geburtswehen nicht [34]. Gesicherte Indikationen der parenteralen Mg-Therapie sind die Neuroprotektion des Fötus bei drohender Frühgeburt, Präeklampsie und Eklampsie. In der Pädiatrie wird dem Mg eine große Rolle bei neurovegetativen Störungen (ADHS) beigemessen [35].

\section{Migräne}

Mg-Mangel wird mit einer erhöhten Neigung für Migräneanfälle in Verbindung gebracht. Als Mechanismen werden u. a. eine Stimulierung der kortikalen Streudepolarisierung, verstärkte Thrombozytenaggregation, Überaktivierung des NMDA-Rezeptors und gesteigerte neurogene Entzündungstendenz diskutiert. Mg-Mangelzustände bei Migränepatienten sind häufig [36]. Gemäß einer Metaanalyse ist die intravenöse Mg-Therapie beim akuten Migräneanfall wirksam sowie orales $\mathrm{Mg}$ als Migräneprophylaxe zur Verringerung der Häufigkeit und Intensität der Anfälle [37]. Beide Therapieformen sollten in das multimodale Therapiekonzept einbezogen werden [37]. Insbesondere orales $\mathrm{Mg}$ stellt dabei eine sichere und gut verträgliche Behandlungsoption dar. Bei Erwachsenen hat sich eine Dosierung von 2-mal täglich 300 mg Mg bewährt. Orales $\mathrm{Mg}$ ist auch für Kinder und Jugendliche empfehlenswert.

\section{Behandlung des Mg-Mangels}

Im Vordergrund steht die orale Therapie. Zum Einsatz kommen Dosierungen von $10-20 \mathrm{mmol}(243-486 \mathrm{mg})$ pro Tag über mehrere Wochen. Im Bedarfsfall kann die Dosis bis zur Durchfallgrenze gesteigert werden. Orales $\mathrm{Mg}$ ist im empfohlenen Dosierungsbereich und bei normaler Nierenfunktion sicher und risikolos. Gelegentlich sind weiche Stühle oder Diarrhö möglich. Üblicherweise gewöhnt sich der Darm an höhere Mg-Mengen, sodass bei Diarrhö nach Dosisreduktion versucht werden sollte, wieder auf die gewünschte Tagesdosis zu steigern. Zum Teil wird die leicht laxierende Wirkung auch als wünschenswert empfunden. Kontraindikationen sind schwere Niereninsuffizienz (GFR <30 ml/min), Exsikkose und Anurie. Die parenterale Therapie beschränkt sich auf spezielle Umstände (z. B. schwerer, sofort auszugleichender Mg-Mangel, Malabsorption, Notfallsituationen) und wird in der Regel intravenös durchgeführt. Mögliches Dosierungsschema: Infusion von 1-2 g Mg-Sulfat(-Heptahydrat) (entsprechend 4$8 \mathrm{mmol}=97-194 \mathrm{mg} \mathrm{Mg}$ ) über 3-6 Stunden, gefolgt von 0,5-1 g Mg-Sulfat(-Heptahydrat) als kontinuierliche Infusion nach Bedarf [38]. Anschließend sollte oral weitertherapiert werden. Spezielle Indikationsgebiete (z. B. Herzrhythmusstörungen, (Prä-) Eklampsie) erfordern die Induktion einer Hypermagnesiämie und damit höhere Mg-Dosen bzw. schnellere Infusionsraten.

\section{Merke}

Die hochdosierte parenterale Therapie sollte dem erfahrenen Kliniker vorbehalten bleiben.

Kontraindikationen sind insbesondere AV-Block, u. a. bradykarde Überleitungsstörungen, Myasthenia gravis und schwere Niereninsuffizienz. Als Überwachungsmaßnahme empfiehlt sich die Prüfung der Patellarsehnenreflexe sowie der Herz-Kreislauf- und Atemfunktion.

\section{Hypermagnesiämie}

Die Hypermagnesiämie beruht meist auf einer Niereninsuffizienz.

\author{
Merke \\ Im Gegensatz zum Mg-Mangel ist die Hypermagnesiämie \\ (Serum-Mg > 1,10 $\mathrm{mmol} / \mathrm{l}$ ) selten.
}

Das Serum-Mg bleibt normalerweise im Referenzbereich, solange die GFR nicht unter $20 \mathrm{ml} / \mathrm{min}$ abfällt. Bei normaler Nierenfunktion lässt sich das Serum-Mg nur durch exzessive Mg-Zufuhr in toxische Bereiche steigern, z. B. durch Mg-haltige Antazida oder Laxanzien. Eine Hypermagnesiämie bleibt bis zu einem Serum-Mg von etwa 2,00 mmol/l asymptomatisch [1]. Je nach Schwere können z. B. Übelkeit, Flush, Müdigkeitserscheinungen, Hypotonie, Hyporeflexie oder Bradykardie auftreten; bei schwerer Hypermagnesiämie (> $5 \mathrm{mmol} / \mathrm{l}$ ) Atemdepression, Herzstillstand und Koma 
[1]. Bei Niereninsuffizienz sind leichte Hypermagnesiämien mit einem Überlebensvorteil assoziiert [39]. Im Fall von Mg-Intoxikation wird als Sofortmaßnahme Kalzium i.v. empfohlen und ggf. eine Hämodialyse.

\section{KERNAUSSAGEN}

- In der Durchschnittsbevölkerung und insbesondere bei Risikogruppen muss mit Magnesiummangel gerechnet werden.

- Eine schlechte Magnesiumversorgung ist ein Risikofaktor für zahlreiche Erkrankungen. Magnesiummangel tritt häufig als Komorbidität auf.

- Einer optimalen Magnesiumversorgung sollte in der Primär-, Sekundär- und Tertiärprävention ein größerer Stellenwert eingeräumt werden.

- Magnesiummangel ist per se therapiebedürftig, da er schwerwiegende Symptome verursachen und zur Exazerbation von Erkrankungen beitragen kann.

- Die orale Magnesiumtherapie ist sicher, unbedenklich und kostengünstig.

\section{Interessenkonflikte}

Oliver Micke: Präsident der Gesellschaft für Magnesium-Forschung e. V. Jürgen Vormann: Vorstand der Gesellschaft für Magnesium-Forschung e. V. Hans-Georg Classen: Vorstand der Gesellschaft für MagnesiumForschung e. V. Klaus Kisters: Vize-Präsident der Gesellschaft für Magnesium-Forschung e. V.

\section{Literatur}

[1] de Baaij JH, Hoenderop JG, Bindels RJ. Magnesium in man: Implications for health and disease. Physiol Rev 2015; 95: 1-46. doi:10.1152/ physrev.00012.2014

[2] Gröber U, Schmidt ], Kisters K. Magnesium in prevention and therapy. Nutrients 2015; 7: 8199-8226. doi:10.3390/nu7095388

[3] Max-Rubner-Institut, Bundesforschungsinstitut für Ernährung und Lebensmittel (Hrsg). Nationale Verzehrsstudie II, Ergebnisbericht Teil 2. 2008: 134-135. im Internet (Stand: 12.12.2019): www.mri.bund.de/file admin/MRI/Institute/EV/NVSII_Abschlussbericht_Teil_2.pdf

[4] Kohlmeier M, Thefeld W, Stelte W et al. Versorgung Erwachsener mit Mineralstoffen und Spurenelementen in der Bundesrepublik Deutschland. In: Kübler W, Anders HJ, Heeschen W, Hrsg. VERA-Schriftenreihe Band V. Niederkleen: Wissenschaftlicher Fachverlag Dr. Fleck; 1995: A23-A27

[5] Schimatschek HF, Rempis R. Prevalence of hypomagnesemia in an unselected German population of 16,000 individuals. Magnes Res 2001; 14: $283-289$

[6] Rosanoff A, Weaver CM, Rude RK. Suboptimal magnesium status in the United States: are the health consequences underestimated? Nutrition Rev 2012; 70: 153-164. doi:10.1111/j.1753-4887.2011.00465.x

[7] Pham PCT, Pharm PMT, Pharm SV et al. Hypomagnesemia in Patients with Type 2 Diabetes. Clin J Am Soc Nephrol 2007; 2: 366-373

[8] Wörwag M, Classen HG, Schumacher E. Prevalence of magnesium and zinc deficiencies in nursing home residents in Germany. Magnes Res 1999; 12: 181-189

[9] Saur P. Magnesium und Sport. Dtsch Z Sportmed 2004; 55: 23-24
[10] Kirschner W, Dudenhausen JW. Anamnese gibt Hinweise auf erhöhtes Risiko für Magnesiummangel. Frauenarzt 2018; 59: 544-548

[11] Elisaf M, Bairaktari E, Kalaitzidis R et al. Hypomagnesemia in alcoholic patients. Alcohol Clin Exp Res 1998; 22: 134

[12] Upala S, Jaruvongvanich V, Wijarnpreecha $\mathrm{K}$ et al. Hypomagnesemia and mortality in patients admitted to intensive care unit: a systematic review and meta-analysis. QJM 2016; 109: 453-459. doi:10.1093/qjmed/hcw048

[13] Ismail Y, Ismail AA, Ismail AA. The underestimated problem of using serum magnesium measurements to exclude magnesium deficiency in adults; a health warning is needed for "normal" results. Clin Chem Lab Med 2010; 48: 323-327. doi:10.1515/CCLM.2010.077

[14] Spätling L, Classen HG, Külpmann WR et al. Diagnostik des Magnesiummangels. Aktuelle Empfehlungen der Gesellschaft für MagnesiumForschung e.V. Fortschritte der Medizin 2000; 118: 49-53

[15] Elin RJ. Assessment of magnesium status for diagnosis and therapy. Magnes Res 2010; 23: S194-S198. doi:10.1684/mrh.2010.0213

[16] DiNicolantonio JJ, O'Keefe JH, Wilson W. Subclinical magnesium deficiency: a principal driver of cardiovascular disease and a public health crisis. Open Heart 2018; 5: e000668. doi:10.1136/openhrt-2017000668

[17] Al Alawi AM, Majoni SW, Falhammar H. Magnesium and Human Health: Perspectives and Research Directions. Int J Endocrinol 2018; 2018: 9041694. doi:10.1155/2018/9041694

[18] Wu J, Xun P, Tang Q et al. Circulating magnesium levels and incidence of coronary heart diseases, hypertension, and type 2 diabetes mellitus: a meta-analysis of prospective cohort studies. Nutr J 2017; 16: 60. doi:10.1186/s12937-017-0280-3

[19] Veronese N, Watutantrige-Fernando S, Luchini C et al. Effect of magnesium supplementation on glucose metabolism in people with or at risk of diabetes: a systematic review and meta-analysis of double-blind randomized controlled trials. Eur J Clin Nutr 2016; 70: 1354-1359. doi:10.1038/ejen.2016.154

[20] von Ehrlich B, Barbagallo M, Classen HG et al. Die Bedeutung von Magnesium für Insulinresistenz, metabolisches Syndrom und Diabetes mellitus - Empfehlungen der Gesellschaft für Magnesium-Forschung e. V. Diabetologie 2014; 9: 96-100. doi:10.1055/s-0034-1366398

[21] Rosanoff A, Plesset MR. Oral magnesium supplements decrease high blood pressure ( $\mathrm{SBP}>155 \mathrm{mmHg}$ ) in hypertensive subjects on antihypertensive medications: a targeted meta-analysis. Magnes Res 2013; 26: 93-99. doi:10.1684/mrh.2013.0343

[22] Cunha AR, D'El-Rei J, Medeiros F et al. Oral magnesium supplementation improves endothelial function and attenuates subclinical atherosclerosis in thiazide-treated hypertensive women. J Hypertens 2017; 35: 89-97. doi: $10.1097 / \mathrm{HJH} .0000000000001129$

[23] Sun X, Zhuang X, Huo M et al. Serum magnesium and the prevalence of peripheral artery disease: The Atherosclerosis Risk in Communities (ARIC) study. Atherosclerosis 2019; 282: 196-201. doi:10.1016/ j.atherosclerosis.2018.12.004

[24] Darooghegi Mofrad M, Djafarian K, Mozaffari H et al. Effect of magnesium supplementation on endothelial function: A systematic review and meta-analysis of randomized controlled trials. Atherosclerosis 2018; 273: 98-105. doi:10.1016/j.atherosclerosis.2018.04.020

[25] Joris PJ, Plat J, Bakker S] et al. Long-term magnesium supplementation improves arterial stiffness in overweight and obese adults: results of a randomized, double-blind, placebo-controlled intervention trial. Am J Clin Nutr 2016; 103: 1260-1266. doi:10.3945/ajcn.116.131466

[26] Shechter M, Bairey Merz CN, Stuehlinger HG et al. Effects of oral magnesium therapy on exercise tolerance, exercise-induced chest pain, and quality of life in patients with coronary artery disease. Am J Cardiol 2003; 91: 517-521. doi:10.1016/s0002-9149(02)03297-6

[27] lezhitsa IN. Potassium and magnesium depletions in congestive heart failure-pathophysiology, consequences and replenishment. Clin Calcium 2005; 15: 123-133. doi:CliCa051118851895 
[28] Adamopoulos C, Pitt B, Sui X et al. Low serum magnesium and cardiovascular mortality in chronic heart failure: a propensitymatched study. Int J Cardiol 2009; 136: 270-277. doi:10.1016/j.ijcard.2008.05.006

[29] Stepura OB, Martynow Al. Magnesium orotate in severe congestive heart failure (MACH). Int J Cardiol 2009; 134: 145-147. doi:10.1016/ j.ijcard.2009.01.047

[30] Branea I, Gaiță D, Drăgulescu I et al. Assessment of treatment with orotate magnesium in early postoperative period of patients with cardiac insufficiency and coronary artery by-pass grafts (ATOMIC). Rom J Intern Med 1999; 37: 287-296

[31] Vierling W, Liebscher DH, Micke O et al. Magnesiummangel und Magnesiumtherapie bei Herzrhythmusstörungen. Empfehlungen der Gesellschaft für Magnesium-Forschung e.V. Dtsch Med Wochenschr 2013; 138: 1165-1171. doi:10.1055/s-0033-1343206

[32] DiNicolantonio J], Liu J, O'Keefe JH. Magnesium for the prevention and treatment of cardiovascular disease. Open Heart 2018; 5: e000775. doi:10.1136/openhrt-2018-000775

[33] Priori SG, Blomstrom-Lundqvist C, Mazzanti A et al. 2015 ESC Guidelines for the management of patients with ventricular arrhythmias and the prevention of sudden cardiac death. Europace 2015; 17: 1601-1687. doi:10.1093/europace/euv319
[34] Spätling L, Classen HG, Kisters K et al. Magnesiumsupplementation in der Schwangerschaft. Empfehlungen der Gesellschaft für MagnesiumForschung e.V. Frauenarzt 2015; 56: 892-897

[35] Liebscher DH, Baerlocher K, Classen HG et al. Magnesiummangel und -therapie bei ADHS. Empfehlungen der Gesellschaft für MagnesiumForschung e.V. Nieren-Hochdruckkrankheiten 2011; 40: 123-128. doi:10.5414/NHP40123

[36] Mauskop A, Varughese J. Why all migraine patients should be treated with magnesium. J Neural Transm 2012; 119: 575-579. doi:10.1007/ s00702-012-0790-2

[37] Chiu HY, Yeh TH, Huang YC et al. Effects of Intravenous and Oral Magnesium on Reducing Migraine: A Meta-analysis of Randomized Controlled Trials. Pain Physician 2016; 19: E97-E112

[38] Picardi S, Lirk P, Blobner M et al. Adjuvanzien in der modernen Anästhesie. Magnesium. Anasthesiol Intensivmed Notfallmed Schmerzther 2015; 50: 380-387. doi:10.1055/s-0041-102214

[39] Xiong J, He T, Wang M et al. Serum magnesium, mortality, and cardiovascular disease in chronic kidney disease and end-stage renal disease patients: a systematic review and meta-analysis. J Nephrol 2019; 32: 791-802. doi:10.1007/s40620-019-00601-6

[40] Van Laecke S. Hypomagnesemia and hypermagnesemia. Acta Clin Belg 2019; 74: 41-47. doi:10.1080/17843286.2018.1516173 